\title{
Marine Benthic Algae of Johnston Atoll: New Species Records, Spatial Distribution, and Taxonomic Affinities with Neighboring Islands ${ }^{1}$
}

\author{
Roy T. Tsuda, ${ }^{2,5}$ Isabella A. Abbott, ${ }^{2,3}$ Peter S. Vroom, ${ }^{4}$ and Fack R. Fisher ${ }^{2}$
}

\begin{abstract}
Forty-five of the 107 species of marine benthic algae collected during 2004 and 2006 NOAA cruises to isolated Johnston Atoll and two additional species from earlier collections represent new species records. Total number of algae is now increased to 189 species: 26 species of cyanobacteria (blue-green algae), 105 species of red algae, 15 species of brown algae, and 43 species of green algae. The macroalga Caulerpa serrulata and the epiphyte Lomentaria bakodatensis were the most widely distributed species at Johnston Atoll based on frequency of occurrence at 10 of 12 stations and 8 of 12 stations, respectively, during the 2004 NOAA cruise. Despite the atoll's isolation, the parasitic red alga Neotenophycus ichtbyosteus and the cyanobacterium Borzia elongata are the only endemic algal species on Johnston Atoll. Nonmetric multidimensional scaling analyses indicate that taxonomic affinities of Johnston Atoll lie between French Frigate Shoals and Wake Atoll. In terms of atolls, biodiversity of the marine flora of Johnston Atoll (i.e., 189 species) is surpassed only by the 256 algal species of the much-larger and better-studied Enewetak Atoll in the Marshall Islands.
\end{abstract}

Johnston Atoll $\left(16^{\circ} 45^{\prime} \mathrm{N}, 169^{\circ} 31^{\prime} \mathrm{W}\right)$ is an isolated atoll (Figure 1) in the North central Pacific that lies $804 \mathrm{~km}$ south of

\footnotetext{
${ }^{1}$ NOAA Coral Reef Conservation Program provided the funds to the Pacific Islands Fisheries Science Center's Coral Reef Ecosystem Division (CRED) for scientific expeditions to the U.S. Pacific Remote Island Areas. Bishop Museum acknowledges CRED for financial support (AB133F07CQ0051) to sort and study the algal specimens from the 2004 and 2006 NOAA cruises to Johnston Atoll. Contribution 2010-002 of the Pacific Biological Survey, Bishop Museum. Manuscript accepted 24 December 2009.

2 Department of Natural Sciences, Bishop Museum, 1525 Bernice Street, Honolulu, Hawai'i 96817.

${ }^{3}$ Department of Botany, St. John Plant Science Building, 3190 Maile Way, University of Hawai'i at Mānoa, Honolulu, Hawai'i 96822.

${ }^{4}$ Joint Institute for Marine and Atmospheric Research, NOAA Fisheries Pacific Islands Fisheries Science Center, Coral Reef Ecosystem Division, 1125B Ala Moana Boulevard, Honolulu, Hawai'i 96814.

${ }^{5}$ Corresponding author (e-mail: roy.tsuda@ bishopmuseum.org).
}

Pacific Science (2010), vol. 64, no. 4:585-605 doi: $10.2984 / 64.4 .585$

(C) 2010 by University of Hawai'i Press

All rights reserved
French Frigate Shoals (Northwestern Hawaiian Islands), 1,448 km northwest of Kingman Reef (Line Islands), and 2,575 km northeast of the Marshall Islands. The atoll has been jointly managed by the U.S. Army, the U.S. Air Force, and the U.S. Fish and Wildlife Service and has served as both a military base and a National Wildlife Refuge. Currently, the lagoon at Johnston Atoll covers an area of $158 \mathrm{~km}^{2}$ and contains a heterogeneous mix of environments ranging from sandy plains to highly rugose coral-dominated reefs. On 6 January 2009, Johnston Atoll and six other U.S. atolls and islands were designated as the Pacific Remote Islands National Marine Monument by presidential decree, which protects and preserves all natural resources within the designated area. Amerson and Shelton (1976) provided extensive information on the natural history of the atoll.

Both land and marine environments at Johnston Atoll have been substantially modified by anthropogenic activities over the past 70 yr (Coles et al. 2001, Lobel 2003). After an initial contract was awarded in 1939 to construct a small navy base, portions of the lagoon were dredged and filled to enlarge Johnston and Sand islets from 0.18 to 2.58 


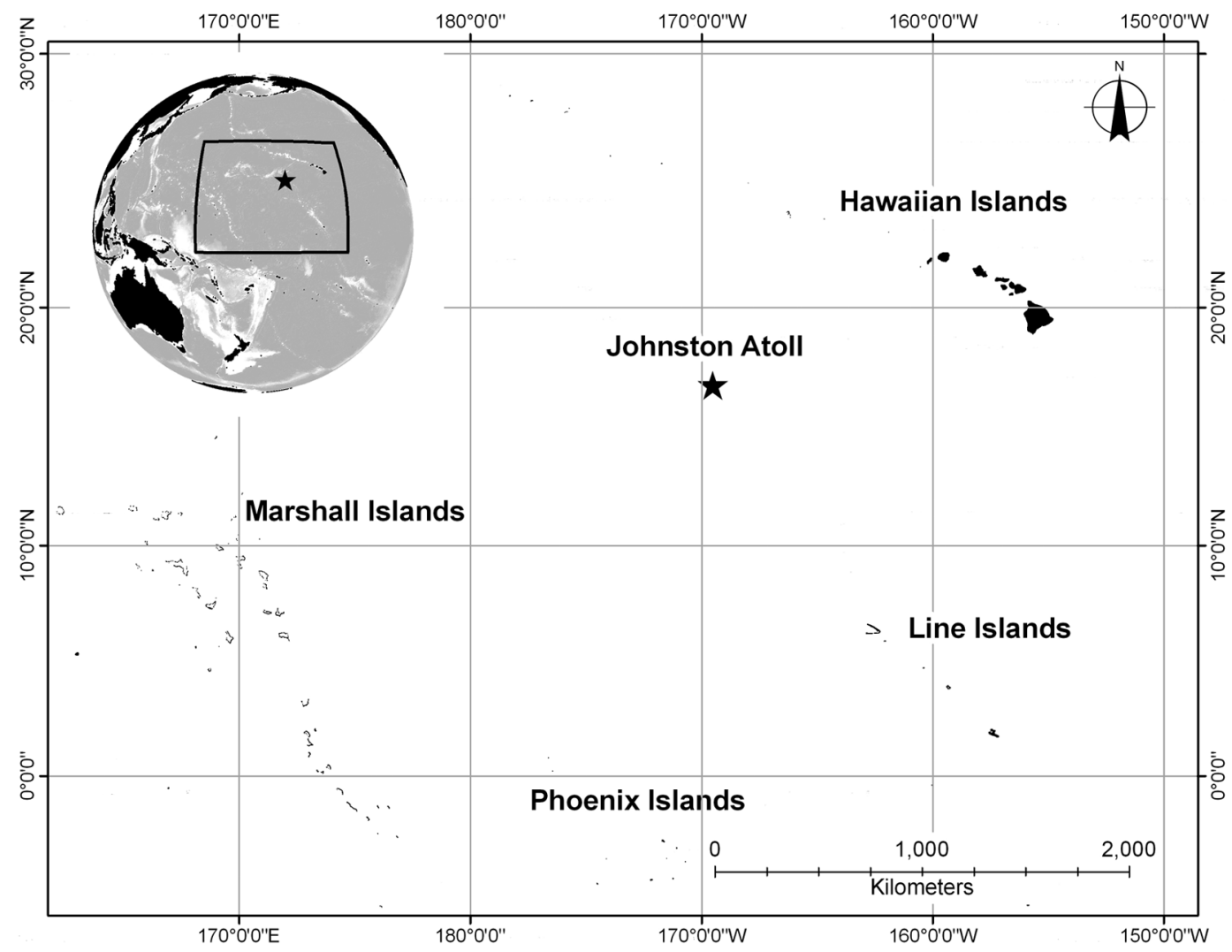

Figure 1. Location of Johnston Atoll in relationship to the Northwestern Hawaiian Islands, the main Hawaiian Islands, the Line Islands, the Phoenix Islands, and the Marshall Islands.

$\mathrm{km}^{2}$ and from 0.04 to $0.09 \mathrm{~km}^{2}$, respectively, and to create two additional islets, North (Akau) $\left(0.1 \mathrm{~km}^{2}\right)$ and East (Hikina) $\left(0.7 \mathrm{~km}^{2}\right)$ islets (Lobel 2003). In August 1958 and July 1962, aboveground thermonuclear devices were detonated over Johnston Atoll that resulted in radioactive fallout on the islets and in the lagoon. Although the atoll is considered to contain relatively healthy reef communities, historical human impacts have undoubtedly affected some marine habitats.

Moul (1964) recorded the first marine alga, Halimeda tuna (Ellis \& Solander) Lamouroux, from Johnston Atoll during his study of the green algae Halimeda and Udotea in the Pacific. Studies were conducted on the ecological impact of dredging operations in the Johnston lagoon (Brock et al. 1965, 1966). As part of that study, Richard G. Bug- geln and Roy T. Tsuda collected marine benthic algae from the lagoon in August and December 1965. The majority of the collections consisted of hammered or chiseled pieces of calcareous substrata covered by algal turf communities. An annotated account of 76 species of marine benthic algae (16 cyanobacteria [blue-green algae], 34 red algae, seven brown algae, and 19 green algae) was provided in an unpublished technical report of the University of Hawai'i, Hawai' $i$ Institute of Marine Biology (Buggeln and Tsuda 1966). The listing also included 16 taxa identified only at the generic level.

Based in part on those collections and earlier collections made by Robert S. Jones in April 1965, Hollenberg $(1968 a, c)$ reported seven species of Polysiphonia: $P$. anomola Hollenberg, P. flaccidissima Hollenberg var. 
decimera Hollenberg [now Neosiphonia flaccidissima (Hollenberg) M. S. Kim \& I. K. Lee], $P$. poko Hollenberg [now Neosiphonia poko (Hollenberg) Abbott], P. scopulorum Harvey var. minima Hollenberg, $P$. setacea Hollenberg, $P$. tenuis Hollenberg [now $P$. triton Silva as a replaced name], and $P$. upolensis (Grunow) Hollenberg, and four species of Herposiphonia: H. pacifica Hollenberg, H. parca Setchell, H. tenella f. secunda (C. Agardh) Hollenberg [now H. secunda (C. Agardh) Ambronn], and $H$. variabilis Hollenberg, from Johnston Atoll. The holotypes of Neosiphonia poko, Polysiphonia triton, and Herposiphonia variabilis were specimens from Johnston Atoll. Corallophila apiculata (Yamada) R. E. Norris was also reported from Johnston Atoll by Hollenberg (1968d) and later by Buggeln and Tsuda (1969) as Centroceras apiculatum Yamada.

R. G. Buggeln and colleagues returned to Johnston Atoll in July 1966 and collected additional algal specimens. The unpublished technical report by Buggeln and Tsuda (1966) was updated to include additional species collected in July 1966 and published (Buggeln and Tsuda 1969). The updated listing included 90 species of marine benthic algae (19 species of cyanobacteria identified by the late "W" Jan Newhouse, 38 species of red algae, nine species of brown algae, and 24 species of green algae) from Johnston Atoll. The red alga Crouania minutissima $\mathrm{Ya}-$ mada was inadvertently listed twice. Fourteen of the 90 entities (four cyanobacteria, two brown algae, and eight red algae) were cited only by the generic names.

Sixteen years later, Aegegian and Abbott (1985) reported seven species of marine algae in waters 45 to $250 \mathrm{~m}$ deep collected during four dives in the manned submersible Maka$l^{i} i$ on the southwestern side of Johnston Atoll in October 1983. The species included two brown algae, Dictyota bartayresiana Lamouroux and Lobophora variegata (Lamouroux) Womersley; four green algae, Caulerpa bikinensis Taylor, Caulerpella ambigua (Okamura) Prud'homme van Reine \& Lokhorst, Dictyosphaeria versluysii Weber-van Bosse, and Halimeda gracilis Harvey; and a red alga of the genus Dasya. Lobophora variegata, C. ambigua, and $D$. vershuysii were previously reported by Buggeln and Tsuda (1969) but not at great depths. A new species of cyanobacteria, Borzia elongata Baker, Patterson \& Ikagawa, was described from Johnston Atoll by Baker et al. (1997).

Coles et al. (2001) reported on the identity of marine species observed and collected during a 5-day marine survey of Johnston Atoll on 16-20 June 2000. A total of 75 algal species was presented (55 red algae, six brown algae, and 14 green algae). An additional 23 algal taxa (16 red algae, three brown algae, and four green algae) were identified to genus. No specimen numbers were provided in the technical report; later, herbarium and slide specimens were provided specimen numbers and deposited in BISH.

Among this collection, a new genus and species of parasitic red alga, Neotonophycus ichthyosteus Kraft \& Abbott, was described growing on its host, Neosiphonia poko (Kraft and Abbott 2002). Holotypes of the parasite and host alga are from Johnston Atoll. Neosiphonia poko is known from several islands and atolls in the Pacific, but the parasite is thus far known only from Johnston Atoll.

The study reported here provides an account of 47 new species records of marine benthic algae from Johnston Atoll, spatial distribution based on frequency of occurrences at 12 stations during the 2004 and 2006 cruises conducted by the National Oceanic and Atmospheric Administration (NOAA), and their taxonomic affinities to algae from neighboring islands and atolls. Prior records of species identified only to the generic level are not included here unless the specimens are the only representatives of the genera from Johnston Atoll (Cladophoropsis sp. and Wurdemannia sp. [Buggeln and Tsuda 1969] and Kallymenia sp. and Spermathamnion sp. [Coles et al. 2001]). Crustose coralline algae were not collected on the two cruises.

\section{MATERIALS AND METHODS}

Fourteen of the 18 NOAA stations (Figure 2) were situated in the lagoon at depths between 1.5 and $17.4 \mathrm{~m}$. The remaining four stations were located on the forereef in waters 13.7 


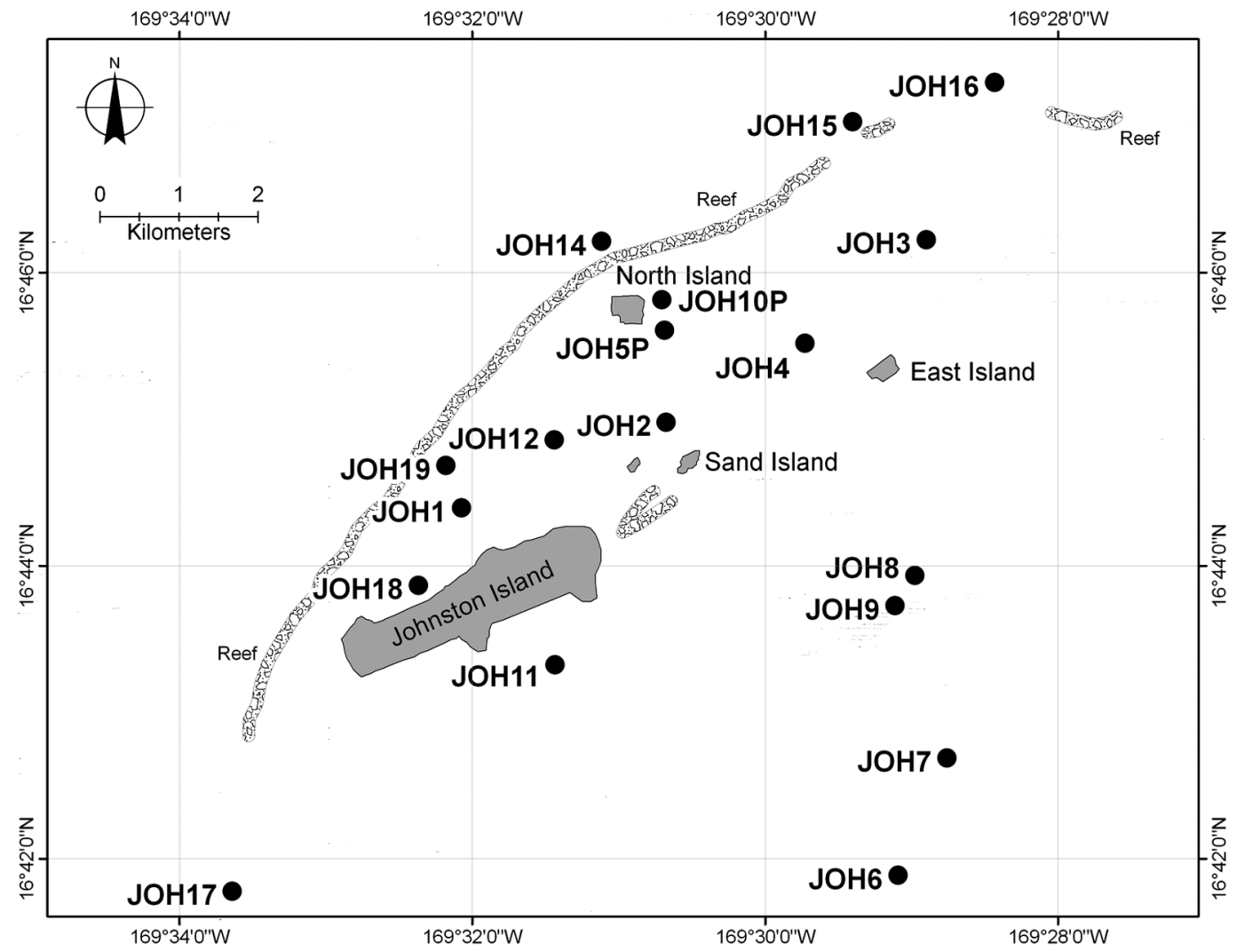

FIgURE 2. Locations of established NOAA monitoring stations at Johnston Atoll. The barrier reef is located on the north and west side of Johnston Island with the south and east side open to the sea.

to $16.8 \mathrm{~m}$ deep; see Preskitt et al. (2004) for sampling method performed during the algal collections. Before examination, plastic bags of frozen algae from each station were thawed in tap water. Thawed seawater was poured carefully out of the bag and replaced with $4 \%$ formalin in seawater. The collections were examined using a dissecting microscope, and all epiphytes and turf were separated. Small specimens $(<1 \mathrm{~cm})$ or sections of larger specimens were mounted on glass slides: specimens were decalcified with $10 \%$ hydrochloric acid, stained with aniline blue, and mounted with $30 \%$ sugar syrup (Karo) with phenol. Macroalgae were mounted on herbarium paper except for one specimen of Amphiroa cf. rigida Lamouroux, which was dried and stored in a vial. Four specimens were also preserved in vials with
$4 \%$ formalin in seawater for future sectioning. The small quantities of nonprocessed replicate specimens from each station were consolidated into separate jars and preserved in $4 \%$ formalin in seawater. The retention of these specimens was to allow us to revisit the site collection, if needed, during critical taxonomic re-examinations of all specimens, including epiphytes.

NOAA stations at Johnston Atoll were identified by using the first three letters of the atoll's name followed by the station and year of collection (e.g., JOH-01-04 signifies Johnston Atoll (JOH), station number 01, and the year 2004). Specimens were collected by Peter S. Vroom and Kimberly N. Page from 12 stations during the 2004 NOAA cruise, and by Peter S. Vroom and Meghan L. Dailer from 18 stations during the 2006 
NOAA cruise. All NOAA specimens were deposited at BISH. Replicate specimens were deposited in the Herbarium of the NOAA Coral Reef Ecosystem Division (CRED), Honolulu. BISH specimen numbers were also inserted in the listing that follows to document the specimens of species cited in Coles et al. (2001). See Appendix 1 for description of NOAA collection sites.

The higher-level classification systems used were as follows: cyanobacteria, Anagnostidis and Komárek (1988), Komárek and Anagnostidis (1989), and Silva et al. (1996); red algae, Abbott (1999) and Choi et al. (2008); green and brown algae, Abbott and Huisman (2004). Citations listed after the species refer to references used in the identification or reexamination of specimens. Annotations were provided when specimens differed from the norm or were uncommon to the central Pacific. New records of algal species for Johnston Atoll are preceded by an asterisk $\left({ }^{*}\right)$.

Because all algal species from each station of the January 2004 and January 2006 NOAA cruises were identified and recorded, the frequency of occurrence (Oosting 1956) (i.e., number or percentage of stations at which a species was recorded) provided one indication of the spatial distribution of a species at Johnston Atoll. In addition, records from the 2004 and 2006 cruises provided two sets of data.

Data on species presence and absence of 404 definitively identified eukaryotic algal species (i.e., red, brown, and green algae) were culled from the literature (French Frigate Shoals, Northwestern Hawaiian Islands [Vroom et al. 2006]; Howland and Baker islands [Tsuda and Trono 1968, Tsuda et al. 2008]; Wake Atoll [Tsuda et al. 2006, 2010]; and Enewetak Atoll, Marshall Islands [Tsuda $1987,2002])$. The data provided a means to compare the marine benthic algal flora of Johnston Atoll with marine floras of five neighboring islands and atolls. A Bray-Curtis similarity matrix of species presence among islands was generated using PRIMER-E software, v. 6 (Clarke and Warwick 2001), and multidimensional scaling (MDS) (number of restarts $=50$ ) was used to map relationships between islands based on floristic composition.
RESULTS

The 107 species collected from Johnston Atoll during the NOAA cruises in January 2004 and January 2006 included 45 new species records (42\%): six cyanobacteria, 25 red algal species, four brown algal species, and 10 green algal species. Two other new species records, Rosenvingea intricata and Codium campanulatum, were ascertained during the reexamination of prior collected specimens that were listed by generic names only. Of the 142 species previously collected from Johnston Atoll, 81 species (57\%) were not recollected during the NOAA cruises: $19 \mathrm{cy}$ anobacteria, 35 red algae, six brown algae, and 21 green algae. Seventy-nine of the 81 species (excluding $R$. intricata and $C$. campanulatum) are listed in Appendix 2. The 189 species of marine benthic algae thus far recorded from Johnston Atoll consist of 26 cyanobacteria, 105 species of red algae, 15 species of brown algae, and 43 species of green algae.

\section{Class Cyanophyceae \\ Order Oscillatoriales \\ Family Oscillatoriaceae}

Lyngbya confervoides C. Agardh; Desikachary (1959):315, pl. 49 (fig. 8), pl. 52 (fig. 1).

Past record: Buggeln and Tsuda (1969). NOAA specimens: JOH-07-04, вIsн 735189; JOH-08-04, вISH 735210.

\section{Family Phormidiaceae}

${ }^{*}$ Hydrocoleum coccineum Gomont; Littler and Littler (2003):276.

NOAA specimen: JOH-19-06, вІен 735532.

*Symploca cf. bydnoides Kützing; Desikachary (1959):335, pl. 60 (figs. 2, 3, 6).

NOAA specimens: JOH-08-06, вISH 735397; JOH-18-06, віsн 735512.

\section{Family Pseudanabaenaceae}

*Leptolyngbya crosbyanum (Tilden) Anagnostidis \& Komárek; Tilden (1910):96, pl. 4 (figs. 60, 61).

NOAA specimens: JOH-01-04, вISH 735038; JOH-02-04, візн 735063; JOH-02- 
06, віSн 735322; JOH-12-04, вISH 735295; JOH-12-06, вІsн 735470.

\section{Order Nostocales}

Family Rivulariaceae

*Calotbrix confervicola (Dillwyn) C. Agardh; Tilden (1910):256, pl. 16 (figs. 6-8).

NOAA specimens: JOH-04-04, віsн 735103 ; JOH-18-06, віSн 735523.

${ }^{*}$ Calotbrix fuscoviolacea P. Crouan \& H. Crouan; Tilden (1910):258, pl. 16 (fig. 10).

NOAA specimen: JOH-10-04, віsн 735269 .

*Dichothrix cf. penicillata Zanardini; Tilden (1910):280.

NOAA specimens: JOH-05P-06, вISH 735358; JOH-10P-06, вISH 735442.

\section{Class Rhodophyceae}

Order Porphyridiales

Family Porphyridiaceae

Stylonema alsidii (Zanardini) K. Drew; Abbott (1999):44, fig. 1B-C.

Past records: Buggeln and Tsuda (1969) as Goniotrichum alsidii (Zanardini) Howe; Coles et al. (2001), віsн 665261. NOAA specimen: JOH-08-04, віsн 735221.

Order Erythropeltidales

Family Erythropeltidaceae

*Erythrotrichia carnea (Dillwyn) J. Agardh; Abbott (1999):45, fig. 1E.

NOAA specimen: JOH-06-04, віsн 735145. Unbranched uniseriate filaments consist of cuboidal cells, $8-10 \mu \mathrm{m}$ diam.

\section{Order Acrochaetiales \\ Family Acrochaetiaceae}

*Acrochaetium bypneae (Børgesen) Børgesen; Abbott (1999):56, fig. 37 as Acrochaetium seriatum Børgesen.

NOAA specimens: JOH-02-04, вISH 735053; JOH-03-04, вISн 735088; JOH-0704, віSн 735173; JOH-08-04, віsн 735207; JOH-12-04, віsн 735308. Filaments are 7-8 $\mu \mathrm{m}$ diam. with monosporangia, $8 \mu \mathrm{m}$ diam., arranged in series.
Order Nemaliales

Family Galaxauraceae

${ }^{*}$ Galaxaura filamentosa Chou; Itono (1980):2, fig. 1.

NOAA specimens: JOH-04-04, вISH 735099; JOH-05P-04, віSн 735129; JOH05P-06, вISH 735353 with dense Polysiphonia coverage; JOH-10P-06, вІІн 735443; JOH19-06, вISH 735537.

\section{Order Gigartinales}

Family Caulacanthaceae

Caulacanthus ustulatus (Turner ex Mertens) Kützing; Abbott (1999):104, figs. 20D-F.

Past record: Coles et al. (2001), вІSн 665789. NOAA specimens: JOH-01-04, вІ्нH 735048; JOH-12-04, вISH 735301.

\section{Family Hypneaceae}

Hypnea spinella J. Agardh; Abbott (1999):117, fig. 25B-E.

Past record: Coles et al. (2001), BISH 665268. NOAA specimens: JOH-01-04, вІІн 735043; JOH-05P-04, віsн 735128; JOH-0704, віsн 735177; JOH-10Р-06, віsн 735445; JOH-11-04, віsн 735278.

\section{Family Peyssonneliaceae}

Peyssonnelia inamoena Pilger; Abbott (1999): 156, fig. $38 B$.

Past record: Coles et al. (2001), BISH 665272. NOAA specimens: JOH-06-04, вISH 735154; JOH-06-06, вІІн 735362; JOH-1104, віsн 735274; JOH-12-04, віsн 735302.

\section{Order Bonnemaisoniales Family Bonnemaisoniaceae}

*Asparagopsis taxiformis (Delile) Trevisan; Abbott (1999):174, fig. 43C-D.

NOAA specimens: JOH-06-06, вISH 735373; JOH-11-04, вISн 735283; JOH-1106, віsн 735452. All specimens were sporophytes (Falkenbergia).

\section{Order Corallinales \\ Family Corallinaceae}

*Amphiroa cf. rigida Lamouroux; Abbott (1999): 178, figs. 44D, 45C. 
NOAA specimens: JOH-08-06, вISH 735418; JOH-09-04, вISH 735245.

*Amphiroa valonioides Yendo; Abbott (1999): 180, figs. $44 E, 45 D$.

NOAA specimens: JOH-03-06, вISH 735336; JOH-05P-06, віsн 735355.

fania pumila Lamouroux; Abbott (1999):189, fig. $48 C$.

Past records: Buggeln and Tsuda (1969) as Jania capillacea Harvey and 7. decussatodichotoma (Yendo) Yendo; Coles et al. (2001), BISH 664286. NOAA specimens: JOH-01-04, вISн 735045; JOH-01-06, віSн 735320; JOH02-04, BISH 735062; JOH-02-06, вISH 735327; JOH-03-06, віsн 735335; JOH-0404, віsн 735098, 735109; JOH-05P-04, віsн 735123; JOH-06-04, віsн 735157; JOH-0704, вISн 735191; JOH-11-06, віsн 725458; JOH-12-04, віsн 735300; JOH-12-06, візн 735465; JOH-18-06, вІsн 735524; JOH-1906, віsн 735536. All branches, decalcified with $\mathrm{HCl}$, were $48-80 \mu \mathrm{m}$ diam.

\section{Order Gelidiales \\ Family Gelidiaceae}

Pterocladiella caloglossoides (Howe) Santelices (1998):243, fig. 3 .

Past record: Coles et al. (2001), віSн 664298. NOAA specimens: JOH-06-04, вIsн 735158; JOH-11-04, візн 735286; JOH-1706, віsн 735508.

\section{Family Gelidiellaceae}

*Gelidiella antipai Celán; Abbott (1999):202, fig. $53 D-F$.

NOAA specimens: JOH-06-06, вISH 735381; JOH-11-04, віsн 735281; JOH-1106, віSн 735456; JOH-18-06, віSн 735522.

\section{Order Rhodymeniales \\ Family Champiaceae}

Champia parvula (C. Agardh) Harvey; Abbott (1999):218, fig. 60A-C.

Past records: Buggeln and Tsuda (1969); Coles et al. (2001), BISH 665772. NOAA specimens: JOH-06-04, вISн 735137; JOH07-04, вISн 735182; JOH-15-06, вISн 735490 .

\section{Family Lomentariaceae}

Lomentaria hakodatensis Yendo; Abbott (1999): 224, fig. 62A-D.

Past records: Buggeln and Tsuda (1969); Coles et al. (2001), віsн 664278. NOAA specimens: JOH-01-04, вISH 735047; JOH02-04, вISн 735056; JOH-06-04, вISн 735134; JOH-06-06, вISH 735379; JOH-0704, віSн 735179; JOH-08-04, віSн 735201; JOH-08-06, вІsн 735398, 735403; JOH-0904, вISн 735239; JOH-09-06, віSн 735423; JOH-11-04, віsн 735288; JOH-12-04, віsн 735297; JOH-16-06, віsн 735501.

\section{Family Rhodymeniaceae}

*Botryocladia skottsbergii (Børgesen) Levring; Abbott (1999):226, fig. 63A-B.

NOAA specimen: JOH-15-06, віsн 735491.

*Chrysymenia okamurae Yamada \& Segawa; Abbott (1999):230, fig. 64C-D.

NOAA specimens: JOH-06-04, вISH 735132; JOH-08-04, вISH 735197; JOH-0904, віsн 735196; JOH-09-06, віsн 735426.

\section{Order Ceramiales \\ Family Callithamniaceae}

Aglaothamnion boergesenii (Aponte \& Ballantine) L'Hardy-Halos \& Rueness; Abbott (1999):244, fig. 67C-F.

Past record: Coles et al. (2001), BISH 665769. NOAA specimen: JOH-06-06, вISн 735375 .

Crouania mageshimensis Itono; Abbott (1999): 293, fig. $82 A-D$.

Past record: Coles et al. (2001), вISH 665809. NOAA specimen: JOH-08-06, вISH 735413. Terminal cells of lateral branches of this species are pointed as opposed to the rounded terminal cells of Crouania minutissima.

Crouania minutissima Yamada; Abbott (1999): 294, fig. 82E-G.

Past record: Buggeln and Tsuda (1969). NOAA specimen: JOH-06-06, віsн 735364. 


\section{Family Ceramiaceae}

Antithamnion antillanum Børgesen; Abbott (1999):248, fig. 69A-B.

Past records: Buggeln and Tsuda (1969); Coles et al. (2001), віsн 665805. NOAA specimens: JOH-02-04, вISH 735055; JOH03-04, вISH 735076; JOH-04-04, вISH 735095; JOH-08-04, віsн 735220; JOH-0806, віSн 735399; JOH-09-04, віSн 735230; JOH-09-06, віSн 735422; JOH-11-04, вISн 735277; JOH-11-06, вІзн 735453; JOH-1606, вISн 735497.

*Antithamnion decipiens (J. Agardh) Athanasiadis; Abbott (1999):250, fig. 69C-D.

NOAA specimen: JOH-07-06, вISH 735387.

Antithamnionella breviramosa (Dawson) Wollaston; Abbott (1999):253, fig. 71A-C.

Past record: Coles et al. (2001), вISH 665240. NOAA specimens: JOH-03-04, вISн 735079; JOH-06-04, вІзн 735144; JOH-0606, віSн 735372; JOH-08-04, віSн 735209; JOH-17-06, віSн 735507.

*Antithamnionella graeffei (Grunow) Athanasiadis; Abbott (1999):254, fig. 71D.

NOAA specimen: JOH-08-06, вISH 735404. Other specimen: вISH 665230, VI16-2000, R. G. DeFelice (not listed in Coles et al. [2001]).

Centroceras minutum Yamada; Abbott (1999): 262, fig. $73 H-I$.

Past record: Coles et al. (2001), вISH 664282. NOAA specimens: JOH-02-04, вISH 735058 ; JOH-02-06, віSн 735324.

Ceramium affine Setchell \& Gardner; South and Skelton (2000):54, figs. 1-10.

Past record: Buggeln and Tsuda (1969). NOAA specimens: JOH-04-04, вIsн 735100; JOH-11-04, вISH 735542.

Ceramium codii (Richards) G. Mazoyer; Cho and Fredericq (2006):496, figs. 24-55.

Past record: Coles et al. (2001), віSн 665784. NOAA specimens: JOH-01-06, вІSн 735321; JOH-02-04, вISH 735060; JOH-0304, віsн 735077; JOH-03-06, віSн 735334 on Microdictyon umbilicatum; JOH-04-04,
вISH 735094; JOH-04-06, вISH 735346; JOH-05P-04, вISH 735118; JOH-10P-04, вISн 735253; JOH-11-04, вІSн 735279; JOH-12-04, вISн 735306; JOH-12-06, вISн 735464; JOH-17-06, віsн 735510; JOH-1906, вISH 735528.

${ }^{*}$ Ceramium krameri South \& Skelton (2000): 69 , figs. $45-51$.

NOAA specimens: JOH-08-04, вISн 735217; JOH-09-04, вISн 735248.

Ceramium macilentum J. Agardh; South and Skelton (2000):71, figs. 52-62.

Past record: Coles et al. (2001), BISH 665273. NOAA specimens: JOH-03-04, вІ्Sн 735083; JOH-06-04, вІІн 735160; JOH-0804, віsн 735200; JOH-08-06, віsн 735419; JOH-09-06, віsн 735431, 735538; JOH-1104, вISH 735284.

Ceramium vagans Silva; Abbott (1999):284, fig. $80 A-D$.

Past records: Buggeln and Tsuda (1969) as Ceramium vagabunda Dawson; Coles et al. (2001), вISH 665237. NOAA specimens: JOH-06-04, вISн 735142; JOH-07-04, вISн 735176; JOH-08-04, віsн 735544; JOH-1206, віSн 735471; JOH-18-06, віsн 735521.

Corallophila apiculata (Yamada) R. E. Norris; Abbott (1999):288, fig. 81A-C.

Past records: Hollenberg (1968d) and Buggeln and Tsuda (1969) as Centroceras apiculatum Yamada; Coles et al. (2001), BISH 665831. NOAA specimens: JOH-03-04, вISH 735084; JOH-05P-04, вISн 735115; JOH-0604, віsн 735139; JOH-06-06, віsн 735374; JOH-08-06, вІSн 735402; JOH-09-04, вІSн 735247; JOH-10P-04, віSH 735256; JOH14-06, вISH 735478. Prostrate and erect axes are $120-180 \mu \mathrm{m}$ diam. with apiculate apices.

Corallophila huysmansii (Weber-van Bosse) R. E. Norris; Abbott (1999):290, fig. 81D-E.

Past records: Buggeln and Tsuda (1969) as Ceramium buysmansii Weber-van Bosse; Coles et al. (2001), віsн 665821. NOAA specimens: JOH-03-06, вІsн 735340; JOH06-04, вISH 735539; JOH-07-04, вISH 735172. Erect axes are $68-120 \mu \mathrm{m}$ diam. and gradually tapering. 
${ }^{*}$ Corallophila itonoi (Ardré) R. E. Norris; Abbott (1999):290, fig. 81F-H.

NOAA specimens: JOH-07-04, вISH 735192; JOH-08-04, вІsн 735226; JOH-1506, вISн 735486. Erect axes, 100-200 $\mu \mathrm{m}$ diam., with apical pinchers present in few axes.

Gayliella flaccida (Kützing) T. O. Cho \& L. Melvor; South and Skelton (2000):65, figs. 32-39, 41-44 as Ceramium flaccidum (Harvey ex Kützing) Ardissone.

Past records: Buggeln and Tsuda (1969) as Ceramium gracillimum var. byssoideum (Harvey) G. Mazoyer; Coles et al. (2001) as $\mathrm{Ce}$ ramium flaccidum (Harvey) Mazoyer, BISH 665242. NOAA specimens: JOH-03-04, вISH 735078; JOH-03-06, віsн 735339; JOH-0604, вISH 735138; JOH-06-06, віSн 735363; JOH-08-04, вISн 735216; JOH-08-06, вІSн 735395; JOH-09-04, вІsн 735246; JOH-0906, вISн 735434; JOH-11-04, віSн 735285; JOH-14-06, віsн 735474; JOH-18-06, вІSн 735515 .

\section{Family Dasyaceae}

*Dasya corymbifera J. Agardh; Abbott (1999): 320, fig. $90 A-C$.

NOAA specimens: JOH-03-04, віSн 735070; JOH-03-06, вІSн 735328; JOH-0404, вISн 735096; JOH-06-04, віSн 735141; JOH-06-06, віsн 735365; JOH-07-04, віSн 735171; JOH-07-06, вІзн 735390; JOH-0804, вISн 735205; JOH-08-06, вISн 735396; JOH-09-04, вISн 735238; JOH-11-06, віsн 735451; JOH-16-06, віSн 735500.

Dasya kristeniae Abbott (1998):105, figs. 1922.

Past record: Coles et al. (2001), віSн 665825. NOAA specimens: JOH-04-04, вISH 735108; JOH-06-04, віsн 735161; JOH-1506, вISH 735487.

Heterosiphonia crispella (C. Agardh) Wynne; Abbott (1999):328, fig. 94A-D.

Past records: Buggeln and Tsuda (1969) as Heterosiphonia wurdemanii var. laxa Børgesen; Coles et al. (2001), віsн 665776. NOAA specimens: JOH-07-04, віsн 735186; JOH10P-06, віsн 735446.

\section{Family Delesseriaceae}

${ }^{*}$ Hypoglossum caloglossoides Wynne \& Kraft; Abbott (1999):337, fig. 97B-C.

NOAA specimens: JOH-05P-04, BISH 735543; JOH-07-04, вISH 735166; JOH10P-06, BISH 735435.

${ }^{*}$ Hypoglossum simulans Wynne, Price \& Ballantine (1989):31, figs. 12-26.

NOAA specimen: JOH-05P-04, вISH 735126.

Taenioma perpusillum (J. Agardh) J. Agardh; Abbott (1999):348, fig. 101A-E.

Past records: Buggeln and Tsuda (1969) as Taenioma macrourum Thuret; Coles et al. (2001), віSн 665815. NOAA specimen: JOH-06-04, вISH 735152.

\section{Family Rhodomelaceae}

Chondria polyrbiza Collins \& Hervey; Abbott (1999):360, fig. $103 G-H$.

Past records: Buggeln and Tsuda (1969) as Chondria repens Børgesen; Coles et al. (2001), s.n. NOAA specimens: JOH-03-04, BISH 735086; JOH-06-04, вілн 735153; JOH-0606, віsн 735370; JOH-08-04, віsн 735202, 735212; JOH-08-06, візн 735408; JOH-0906, вISн 735430; JOH-10P-04, вISн 735262; JOH-14-06, віsн 735473; JOH-15-06, віsн 735488; JOH-19-06, віSH 735530. Erect branches are 160-200 $\mu \mathrm{m}$ diam. with mature cortical cells rectilinear.

*Chondria simpliciuscula Weber-van Bosse; Abbott (1999):361, fig. 104 $A-F$.

NOAA specimens: JOH-01-04, вISH 735050; JOH-03-06, вISH 735333; JOH05P-04, вISH 735124; JOH-06-04, вISH 735148; JOH-06-06, вISн 735376; JOH-0704, візн 735180, 735181; JOH-07-06, віSн 735384; JOH-08-04, вілн 735203; JOH-0806, віsн 735401; JOH-10Р-06, віsн 735439; JOH-15-06, віsн 735493; JOH-16-06, віSн 735504; JOH-17-06, віSH 735505. Erect branches are $240-320 \mu \mathrm{m}$ diam. with distal cortical cells hexagonal or elongate ovoid.

${ }^{*}$ Herposiphonia obscura Hollenberg (1968c): 549, fig. 25.

NOAA specimens: JOH-03-06, вISH 
735337; JOH-10P-04, віSн 735267; JOH10P-06, вISн 735444.

Herposiphonia pacifica Hollenberg (1968c):549, figs. $2 A, 2 B, 4,19$.

Past record: Hollenberg (1968c). NOAA specimen: JOH-10P-04, вISH 735257.

Herposiphonia parca Setchell; Hollenberg (1968c):552, figs. $2 C, 16,20,22,23$.

Past records: Hollenberg (1968c); Coles et al. (2001), віsн 665778. NOAA specimens: JOH-04-04, вISн 735106; JOH-06-04, вІІн 735159; JOH-06-06, вІзн 735371; JOH-0804, віsн 735208.

Herposiphonia secunda (C. Agardh) Ambronn; Abbott (1999):376, fig. 109A-E.

Past records: Hollenberg (1968c) as $H$. tenella (C. Agardh) Ambronn f. secunda (C. Agardh) Hollenberg; Coles et al. (2001), BISH 665793. NOAA specimen: JOH-06-04, вІ вн 735143 .

Herposiphonia variabilis Hollenberg (1968c): 557, figs. $1 F, 2 G, 17,18,21$.

Past records: Hollenberg (1968c) (holotype); Coles et al. (2001), віsн 664349. NOAA specimens: JOH-03-04, вISн 735081; JOH-07-04, віsн 735183; JOH-08-04, візн 735213 ; JOH-12-04, вISH 735294.

Laurencia majuscula (Harvey) Lucas; Saito (1969):149; Abbott (1999):388, fig. 112G-H.

Past record: Coles et al. (2001), вISH 664341. NOAA specimens: JOH-03-04, вІSн 735085; JOH-06-04, віSн 735136. Immature fragment is less than $0.7 \mathrm{~mm}$ long with no lenticular thickenings and with faint protruding cortical cells.

Neosiphonia flaccidissima (Hollenberg) M. S. Kim \& I. K. Lee; Hollenberg (1968a):63 as Polysiphonia flaccidissima Hollenberg.

Past record: Hollenberg (1968a) as Polysiphonia flaccidissima Hollenberg. NOAA specimens: JOH-08-06, віsн 735546; JOH-09-04, BISH 735545 .

Neosiphonia poko (Hollenberg) Abbott; Hollenberg (1968a):70, fig. $3 A$ as Polysiphonia poko Hollenberg.

Past record: Hollenberg (1968a) (holotype); Kraft and Abbott (2002). Other speci- men: вISH 664337, VI-17-2000, R. G. DeFelice (not listed in Coles et al. [2001]).

Neosiphonia sphaerocarpa (Børgesen) M. S. Kim \& I. K. Lee; Hollenberg (1968a):87 as Polysiphonia sphaerocarpa Børgesen.

Past record: Coles et al. (2001), BISH 664334. NOAA specimen: JOH-07-04, вІзн 735194.

*Neosiphonia tepida (Hollenberg) S. M. Guimarães \& M. T. Fujii; Hollenberg (1968b):205, fig. 3D-E as Polysiphonia tepida Hollenberg.

NOAA specimens: JOH-01-04, вISH 735044; JOH-01-06, вISн 735313; JOH-0304, віsн 735082; JOH-03-06, віSн 735341; JOH-08-06, віsн 735400; JOH-09-06, візн 735425; JOH-10Р-04, віsн 735252; JOH11-04, BISH 735287; JOH-12-06, вISH 735462; JOH-19-06, віsн 735527, 735531. Erect axes, 96-140 $\mu \mathrm{m}$ diam., with seven pericentral cells.

Polysiphonia anomala Hollenberg (1968a):59, fig. $1 A-C$.

Past record: Hollenberg (1968a). NOAA specimens: JOH-06-04, вISH 735149; JOH06-06, вISн 735366; JOH-08-04, вISH 735204; JOH-08-06, вISн 735414; JOH-0906, 735433; JOH-12-04, віsн 735309; JOH14-06, вілн 735476, 735483.

Polysiphonia delicatula Hollenberg (1968a):62, fig. $1 F$.

Past record: Coles et al. (2001), BISH 665244. NOAA specimens: JOH-01-06, вISH 735312; JOH-03-04, вISн 735075; JOH-05P04, вISн 735127; JOH-05P-06, вISн 735361; JOH-06-04, вISн 735131; JOH-06-06, вISн 735378 on Peyssonnelia sp.; JOH-07-06, вІ вн 735386; JOH-08-04, вISH 735211.

Polysiphonia exilis Harvey; Hollenberg (1968b):200, figs. $1 C, 3 G$.

Past record: Coles et al. (2001), BISH 664317. NOAA specimens: JOH-04-06, віSн 735343; JOH-08-04, віsн 735224; JOH-0904, вISн 735242; JOH-12-04, віSн 735299. Erect axes, 100-120 $\mu \mathrm{m}$ diam., with 7-9 pericentral cells.

*Polysiphonia bomoia Setchell \& Gardner; Hollenberg (1968b):201, fig. $2 B$. 
NOAA specimen: JOH-11-04, вISH 735290.

Polysiphonia scopulorum Harvey; Hollenberg (1968a):79.

Past record: Hollenberg (1968a); Coles et al. (2001), вISH 665798. NOAA specimens: JOH-02-04, віsн 735059; JOH-08-04, віsн 735222. Rhizoid is connected to the pericentral cell by a pit connection.

Polysiphonia setacea Hollenberg (1968a):85, fig. 5A-C.

Past record: Hollenberg (1968a). NOAA specimens: JOH-05P-06, BISH 735351; JOH10-06, вISн 735450.

*Polysiphonia cf. tsudana Hollenberg (1968b): 205, figs. $1 F, 1 G, 2 C$.

NOAA specimen: JOH-16-06, вISH 735499 (sterile). Erect axes, 142-160 $\mu \mathrm{m}$ diam., are larger than the epizoic type specimens (45-50 $\mu \mathrm{m}$ diam.). Prostrate axes and base of erect axes consist of four pericentral cells with upper erect axes consisting of 6-7 pericentral cells.

Polysiphonia upolensis (Grunow) Hollenberg (1968a):94, figs. 6D-E, 29, 35, 42.

Past record: Hollenberg (1968a). NOAA specimens: JOH-10P-04, BISH 735263; JOH11-04, віsн 735280. Branched erect axes, 96$132 \mu \mathrm{m}$ diam., with swollen rhizoids.

\section{Family Sarcomeniaceae}

${ }^{*}$ Dotyella hawaiiensis (Doty \& Wainwright) Womersley \& Shepley; Abbott (1999):334, fig. $96 A-C$.

NOAA specimens: JOH-03-04, вISH 735541; JOH-05P-06, BISH 735360; JOH07-04, BISH 735185.

*Malaconema minimum Hollenberg (1963): 169, figs. 1-3.

NOAA specimens: JOH-01-04, вISH 735042; JOH-12-06, віsн 735469; JOH-1906, віSн 735529.

\section{Family Wrangeliaceae}

Anotrichium secunda (Harvey ex J. Agardh) Furnari; Abbott (1999):245, fig. 68A-C.

Past record: Coles et al. (2001), віSH 665819. NOAA specimens: JOH-03-04, віsн
735072; JOH-06-04, вISн 735133; JOH-0704, віSн 735168; JOH-07-06, віSн 735389; JOH-08-06, віsн 735406; JOH-09-04, віsн 735241; JOH-09-06, віsн 735432; JOH-1406, віSн 735475; JOH-16-06, віSн 735503.

Anotrichium tenue (C. Agardh) Nägeli; Abbott (1999):247, fig. 68D.

Past records: Buggeln and Tsuda (1969) as Griffithsia tenuis C. Agardh; Coles et al. (2001), віsн 665785. NOAA specimens: JOH-06-06, віsн 735369; JOH-08-04, віsн 735206.

Griffithsia beteromorpha Kützing; Abbott (1999):300, fig. 84A-C.

Past record: Coles et al. (2001), BISH 664297. NOAA specimens: JOH-01-04, вISH 735052; JOH-02-04, вISH 735057; JOH-0206, вISн 735325; JOH-04-04, вISн 735102; JOH-05P-04, вISн 735125; JOH-08-04, віSн 735225; JOH-08-06, вISн 735415; JOH-10P04, віsн 735254; JOH-10P-06, вISн 735448.

Griffithsia subcylindrica Okamura; Abbott (1999):302, fig. 85C-D.

Past record: Coles et al. (2001), віSH 664342. NOAA specimens: JOH-06-04, віsн 735156; JOH-07-04, вISH 735188; JOH-10P04, вISн 735540.

*Haloplegma duperreyi Montagne; Abbott (1999):305, fig. 86E.

NOAA specimen: JOH-07-04, вISH 735165 .

${ }^{*}$ Lejolisea pacifica Itono; Abbott (1999):307, fig. $86 F-G$.

NOAA specimens: JOH-09-04, вISH 735244 on Halimeda taenicola. Filaments include a single stalked tetrasporangium, $8 \mu \mathrm{m}$ diam.

Ptilothamnion cladophorae (Yamada \& Tanaka) Feldmann-Mazoyer; Abbott (1999):313, fig. $87 I$.

Past record: Coles et al. (2001), BISH 665263. NOAA specimens: JOH-01-06, віsн 735319; JOH-03-04, вISн 735073; JOH-05P04, вISн 735119; JOH-05P-06, вISн 735356; JOH-06-04, віSн 735151; JOH-07-06, віSн 735383; JOH-10P-04, вISH 735261; JOH10P-06, віSн 735436; JOH-12-06, вISн 735467; JOH-19-06, віsн 735526. 
Spermothamnion sp.

Past record: Coles et al. (2001), BISH 664305. NOAA specimen: JOH-10P-06, BISH 735266 (sterile). Coles et al. (2001) listed this specimen as Spermothamnion sp.; the NOAA specimen (BISH 735266) is sterile and could be either a Spermothamnion or Tiffaniella.

Class Phaeophyceae

Order Ectocarpales

Family ECTOCARPACEAE

${ }^{*}$ Kuetzingiella elachistaeformis (Heydrich) Balakrishnan \& Kinkar; Abbott and Huisman (2004):163, fig. 60A-B.

NOAA specimen: JOH-11-04, віSн 735282.

Order Scytosiphonales

Family Scytosiphonaceae

${ }^{*}$ Rosenvingea intricata (J. Agardh) Børgesen; Abbott and Huisman (2004):185, fig. 69A-C.

Past record: Coles et al. (2001) as Rosenvingea sp., віsн 665791, VI-18-2000, leg. R. G. DeFelice.

Order Sphacelariales

Family Sphacelariaceae

Sphacelaria novaebollandiae Sonder; Tsuda (1972):93, pl. 1 (fig. 5).

Past records: Buggeln and Tsuda (1969), Coles et al. (2001), віSн 665797. NOAA specimens: JOH-15-06, вISH 735489; JOH18-06, вISH 735520.

Sphacelaria rigidula Kützing; Abbott and Huisman (2004):190, fig. 72C.

Past record: Buggeln and Tsuda (1969) as Sphacelaria furcigera Kützing. NOAA specimen: JOH-06-04, віsн 735146.

\section{Order Dictyotales}

Family Dictyotaceae

Dictyopteris repens (Okamura) Børgesen; Tsuda (1972):94, pl. 3 (fig. 1).

Past record: Coles et al. (2001), вISH 664294. NOAA specimens: JOH-08-04, вISH 735218; JOH-11-04, вISH 735275; JOH-1806, вISH 735519.
Dictyota ceylanica Kützing; Abbott \& Huisman (2004):202, fig. 77B.

Past records: Coles et al. (2001) as Dictyota divaricata Lamouroux, BISH 664292, and as D. acutiloba J. Agardh, BISH 665764. NOAA specimens: JOH-07-04, вISн 735174; JOH10P-04, вISн 735265; JOH-11-04, вISн 735273.

*Dictyota friabilis Setchell; Tsuda (1972):96, pl. 4 (fig. 3).

NOAA specimens: JOH-02-04, BISH 735065; JOH-06-04, вІІн 735150; JOH-0704, вІSн 735170; JOH-14-06, віSн 735479; JOH-15-06, вІSн 735492; JOH-18-06, вISн 735514.

*Dictyota sandvicensis Sonder; Abbott and Huisman (2004):205, fig. 78 $A-B$.

NOAA specimens: JOH-01-04, віsн 734049; JOH-07-06, віsн 735391; JOH-1104, віsн 735276; JOH-17-06, віsн 735506.

Lobophora variegata (Lamouroux) Womersley ex Oliveira; Taylor (1950):97 as Pocockiella variegata (Lamouroux) Papenfuss.

Past records: Buggeln and Tsuda (1969) as Pocockiella variegata (Lamouroux) Papenfuss; Aegegian and Abbott (1985), $140 \mathrm{~m}$ deep; Coles et al. (2001), вISH 665788. NOAA specimens: JOH-03-04, вISн 735080; JOH03-06, вISH 735332; JOH-04-04, вISH 735097; JOH-06-04, вISH 735155; JOH-0704, вISH 735178; JOH-08-04, вISH 735198; JOH-08-06, вІSн 735411; JOH-09-04, вІІн 735249; JOH-11-04, віsн 735271; JOH-1506, візн 735485; JOH-16-06, вілн 735498; JOH-17-06, вISн 735509.

\section{Order Cutleriales \\ Family Cutleriaceae}

${ }^{*}$ Cutleria irregularis Abbott \& Huisman (2003):175, figs. 7-15.

NOAA specimens: JOH-01-04, BISH 735046; JOH-01-06, віsн 735317; JOH-0204, віsн 735061; JOH-02-06, віsн 735326; JOH-04-06, віsн 735345; JOH-05P-04, віsн 735120; JOH-05P-06, віsн 735354; JOH-0706, віsн 735385; JOH-10Р-04, віsн 735259; JOH-11-06, віsн 735455; JOH-12-04, віsн 735293; JOH-12-06, вілн 735461; JOH-1806, віSн 735516; JOH-19-06, віSн 735535. 


\section{Class Ulvophyceae Order Ulvales \\ Family Ulvaceae}

Ulva clathrata (Roth) C. Agardh; Abbott and Huisman (2004):46, fig. 5A-C as Enteromorpha clathrata (Roth) Greville.

Past record: Coles et al. (2001) as Enteromorpha clathrata, вISH 665781. NOAA specimens: JOH-04-04, вISH 735104; JOH-05P04, віSн 735117; JOH-07-04, віSн 735193; JOH-08-06, віsн 735409; JOH-10P-04, віsн 735264; JOH-12-04, вISн 735304. All specimens are less than $5 \mathrm{~mm}$ long and possess mainly uniseriate branches from base to apex.

\section{Class Cladophorophyceae \\ Order Cladophorales \\ Family Anadyomenaceae}

Microdictyon setchellianum Howe; Abbott and Huisman (2004):61, fig. 15A.

Past record: Buggeln and Tsuda (1969). NOAA specimen: JOH-08-06, вIsн 735394.

*Microdictyon umbilicatum (Velley) Zanardini; Abbott and Huisman (2004):62, fig. 15B.

NOAA specimens: JOH-03-04, вISH 735090; JOH-03-06, віsн 735329; JOH-0806, вISн 735548.

\section{Family Cladophoraceae}

*Cladophora dotyana Gilbert; Abbott and Huisman (2004):71, fig. 20A-B.

NOAA specimens: JOH-11-04, вISH 735289; JOH-11-06, вISH 735454.

${ }^{*}$ Cladophora cf. Alexuosa (O. F. Müller) Kützing; Abbott and Huisman (2004):72, fig. 20C.

NOAA specimens: JOH-05P-04, BISH 735114; JOH-05P-06, вISн 735349.

*Cladophora luxurians (Gilbert) Abbott \& Huisman; Abbott and Huisman (2004):77, fig. $22 D$.

NOAA specimens: JOH-01-04, вІSн 735040; JOH-07-04, вISH 735190.

\section{Family Siphonocladaceae}

\section{Cladophoropsis sp.}

Past record: Buggeln and Tsuda (1969).

NOAA specimen: JOH-12-04, вISн 735303.
Dictyosphaeria versluysii Weber-van Bosse; Egerod (1952):351, figs. 1a, $2 b-k$.

Past record: Buggeln and Tsuda (1969); Aegegian and Abbott (1985), $50 \mathrm{~m}$ deep; Coles et al. (2001), BISH 665257. NOAA specimens: JOH-01-06, вІsн 735311; JOH04-04, вISн 735093; JOH-04-06, вISн 735344; JOH-05P-04, вISн 735113; JOH05P-06, вISн 735359; JOH-08-04, вISн 735214; JOH-08-06, віSн 735412; JOH10P-06, віSн 735437; JOH-14-06, віSн 735480; JOH-18-06, віsн 735513; JOH-1906, вISH 735534.

\section{Family Valoniaceae}

Valonia ventricosa J. Agardh; Kraft (2007):121, fig. 51A-D.

Past record: Buggeln and Tsuda (1969). NOAA specimens: JOH-01-06, вISH 735310; JOH-04-04, віsн 735092; JOH-04-06, вІSн 735348; JOH-05P-04, вISH 735112; JOH05P-06, вISH 735352; JOH-10P-04, вISH 735250; JOH-10P-06, вISH 735441; JOH12-04, вISн 735296; JOH-12-06, вISн 735466; JOH-19-06, вISH 735533.

\section{Class Bryopsidophyceae Order BRYopsidales Family Bryopsidaceae}

Bryopsis pennata Lamouroux; Egerod (1952): 370, fig. 7.

Past records: Buggeln and Tsuda (1969), Coles et al. (2001) as B. pennata, вISH 663163 and as $B$. bypnoides, BISH 663164. NOAA specimens: JOH-07-04, вISH 735167; JOH11-04, вISн 735270; JOH-11-06, вISH 735459. Coles et al. (2001) described the bloom of Bryopsis bypnoides Lamouroux, up to $10 \mathrm{~cm}$ long, at the sewer outfall site on the south side of Johnston Islet during June 2000. The specimens, however, are more applicable to $B$. pennata.

\section{Family Caulerpaceae}

Caulerpa bikinensis Taylor (1950):66, pl. 33.

Past record: Aegegian and Abbott (1985), 45-76 (121) $m$ deep. NOAA specimens: JOH-07-04, віSн 735175; JOH-07-06, вІІн 735393; JOH-08-04, віsн 735223; JOH-08- 
06, вISH 735405; JOH-09-04, вISH 735236; JOH-09-06, віsн 735427.

*Caulerpa nummularia Harvey ex J. Agardh; Abbott and Huisman (2004):121, fig. $44 A$.

NOAA specimens: JOH-07-04, віsн 735169; JOH-08-04, віsн 735229.

Caulerpa racemosa (Forsskål) J. Agardh; Abbott and Huisman (2004):122, fig. 44B-C.

Past records: Buggeln and Tsuda (1969); Coles et al. (2001), віsн 663165. NOAA specimen: JOH-08-04, віsн 735215.

Caulerpa serrulata (Forsskål) J. Agardh; Meñez and Calumpong (1982):9, pl. $2 E$.

Past record: Coles et al. (2001), вISH 664350. NOAA specimens: JOH-02-04, вISH 735064; JOH-03-04, вІІн 735087; JOH-0404, віSн 735107; JOH-04-06, вISн 735342; JOH-05P-04, вISH 735111; JOH-06-04, вISH 735130; JOH-06-06, вISн 735368; JOH-0704, віSн 735162; JOH-07-06, віSн 735392; JOH-08-04, віsн 735219; JOH-08-06, віsн 735416; JOH-09-04, вІІн 735233; JOH-0906, віsн 735421; JOH-10P-04, віSн 735251; JOH-10P-06, віsн 735438; JOH-12-04, віsн 735292; JOH-14-06, віsн 735482; JOH-1606, віSн 735496; JOH-18-06, віSн 735511; JOH-19-06, віsн 735525.

${ }^{*}$ Caulerpa taxifolia (Vahl) C. Agardh; Abbott and Huisman (2004):124, fig. 46A-B.

NOAA specimens: JOH-07-04, вISH 735164; JOH-07-06, вІІн 735382; JOH-0804, вISH 735228; JOH-09-04, вISH 735234; JOH-09-06, віSн 735420.

Caulerpa webbiana Montagne; Meñez and Calumpong (1982):10, fig. 20G-7.

Past record: Coles et al. (2001), віsн 665775. NOAA specimens: JOH-06-06, вISH 735380; JOH-15-06, віsн 735484.

\section{Family Codiaceae}

${ }^{*}$ Codium campanulatum P. C. Silva \& M. E. Chacana; Abbott and Huisman (2004):102, fig. $35 C-D$.

Past record: Buggeln and Tsuda (1969) as Codium sp., reexamination of RT $1127 \mathrm{~b}$ (вISH 711933), RT 1174 (віSн 711931), RT 1556 (віSн 711930). Fragments, up to 15 $\mathrm{mm}$ long, possess bell-shaped utricles.
Family Derbesiaceae

Derbesia cf. fastigiata Taylor; Abbott and Huisman (2004):128, fig. 48 $A-B$.

NOAA specimen: JOH-02-04, BISH 735054. Sterile siphons are $10 \mu \mathrm{m}$ diam. with terminal dichotomous branches.

\section{Family Halimedaceae}

*Halimeda taenicola W. R. Taylor (1950):86, pl. 46 (fig. 1); Hillis (1959):354, pl. 2 (fig. 6), pl. 5 (fig. 12), pl. 6 (fig. 14), pl. 11 .

NOAA specimens: JOH-03-04, вISH 735071; JOH-03-06, вISн 735330; JOH-0404, вISH 735110; JOH-06-04, вISH 735135; JOH-06-06, вІІн 735367; JOH-07-04, вІІн 735163; JOH-07-06, візн 735388; JOH-0804, віSн 735199; JOH-08-06, віSн 735407; JOH-09-04, віSн 735232; JOH-09-06, віSн 735429; JOH-14-06, вілн 735472; JOH-1506, віSн 735495; JOH-16-06, віsн 735502.

\section{Family Udoteaceae}

${ }^{*}$ Rhipidosiphon javensis Montagne; Abbott and Huisman (2004):140, fig. 52C-D.

NOAA specimens: JOH-01-06, вІ вн 735316; JOH-09-04, вISH 735235.

*Pseudochlorodesmis abbreviata Gilbert; Abbott and Huisman (2004):141, fig. $53 \mathrm{~A}$ as Siphonogramen abbreviata (Gilbert) Abbott \& Huisman.

NOAA specimens: JOH-09-04, вISH 735243; JOH-18-06, вISH 735517. As per Verbruggen et al. (2009), the genus Pseudochlorodesmis is used instead of Siphonogramen.

Pseudochlorodesmis parva Gilbert; Abbott and Huisman (2004):142, fig. 53B-C as Siphonogramen parva (Gilbert) Abbott \& Huisman.

Past record: Buggeln and Tsuda (1969). NOAA specimen: JOH-15-06, вIsн 735494.

\section{Class Dasycladophyceae Order Dasycladales Family Dasycladaceae}

*Neomeris bilimbata J. Koster; Kraft (2007): 292, fig. 108.

NOAA specimen: JOH-14-06, BISH 735477. 
TABLE 1

Frequency (6/12 or $50 \%$ and greater) of Algal Species at Stations 1-12 during January 2004 and January 2006 NOAA Cruises to Johnston Atoll

\begin{tabular}{lrr}
\hline \hline & \multicolumn{2}{c}{$\begin{array}{c}\text { Frequency } \\
\text { (12 Stations) }\end{array}$} \\
\cline { 2 - 3 } Species & 2004 & 2006 \\
\hline Caulerpa serrulata & 10 & 6 \\
Lomentaria bakodatensis & 8 & 4 \\
Jania pumila & 7 & 6 \\
Ceramium codii & 7 & 4 \\
Lobophora variegata & 7 & 3 \\
Halimeda taenicola & 6 & 5 \\
Dasya corymbifera & 6 & 5 \\
Antithamnion antillarum & 6 & 3 \\
Griffithsia heteromorpha & 6 & 3 \\
Cutleria irregularis & 5 & 6 \\
Chondria simpliciuscula & 5 & \\
\hline
\end{tabular}

\section{DISCUSSION}

Eleven algal species (Table 1) were recorded at six $(50 \%)$ or more of 12 stations (stations 1-12) during either the 2004 or 2006 cruise. Four species can be considered macroalgae: two green algae (Caulerpa serrulata and Halimeda taenicola) and two brown algae (Lobophora variegata and Cutleria irregularis). The remaining seven red algal species were turf or epiphytes, less than $1 \mathrm{~cm}$ long. The macroalga Caulerpa serrulata and the epiphyte Lomentaria hakodatensis were the most spatially distributed species at Johnston Atoll. Only two species, Caulerpa serrulata and Jania pumila, were recorded in $50 \%$ or more of the 12 stations during both NOAA cruises.

The isolation and location of Johnston Atoll in the central Pacific should favor a setting for endemism. Yet, the only endemic algal species of the 189 species recorded from Johnston Atoll are the parasitic red alga Neotenophycus ichthyosteus (Kraft and Abbott 2002) and the cyanobacterium Borzia elongata (Baker et al. 1997), which will most likely be found at other islands if the parasite's host, Neosiphonia poko, and cyanobacteria are critically examined. Maragos and Jokiel (1986), likewise, did not find endemism among the 33 species of stony corals at Johnston Atoll, which had the least coral diversity compared with Hawai'i (45 species), Phoenix Islands (85 species), Line Islands (70 species), and Marshall Islands (366 species).

Gosline (1955) considered the 158 species of inshore fish fauna at Johnston Atoll an extension of the Hawaiian fish fauna. Based on an updated listing of 271 species of fishes from Johnston Atoll, Randall et al. (1985) reported the fish fauna impoverished as compared with those of the Hawaiian Islands (680 species) and the Marshall Islands (817 species). Reasons for the depauperate fish fauna have been attributed to the atoll's isolation, small size, and paucity of marine habitats. Three unidentified fish species observed in waters deeper than $100 \mathrm{~m}$ (Randall et al. 1985) and the deepwater Centropyge nabackyi Kosaki (1989) may be the only endemic fish species at Johnston Atoll. Kosaki et al. (1991) concluded that repeated extinctions and recolonization from the nearest high-island refuge rather than long-term residence of species may reflect the Hawaiian influence on the current fish fauna (301 species) of Johnston Atoll.

Nonmetric multidimensional scaling analyses (Figure 3) indicate that the taxonomic affinities of eukaryotic marine benthic algae of Johnston Atoll lie between its nearest northern neighbor French Frigate Shoals (Northwestern Hawaiian Islands) and Wake

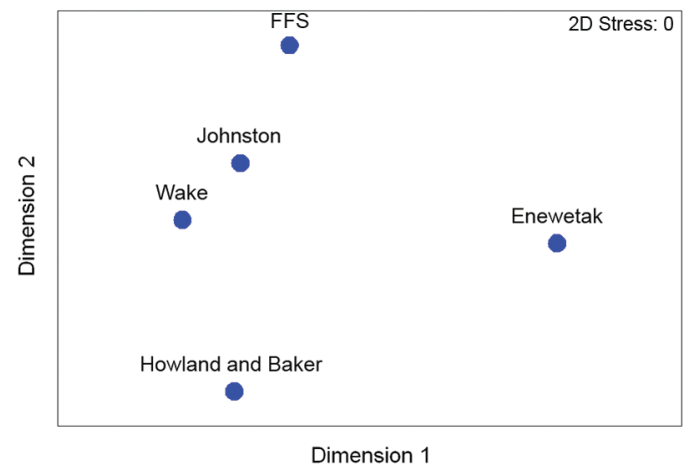

Figure 3. Nonmetric multidimensional scaling plot comparing Johnston Atoll with neighboring islands and atolls based on presence and absence of marine eukaryotic algal species: French Frigate Shoals (FFS), Howland and Baker islands, Wake Atoll, and Enewetak Atoll. 
Atoll to the west. Past studies cited in this paper have hypothesized that Johnston Atoll represents the "bridge" that links the Hawaiian Archipelago to other islands and atolls in the Pacific. Johnston Atoll and French Frigate Shoals contain 160 and 176 eukaryotic algal species, respectively. Of these, 82 are shared by both islands.

Of the six islands included in this analysis, 18 species (three species of green algae and 15 species of red algae) are unique to Johnston Atoll and French Frigate Shoals. The green algae include Derbesia fastigiata, Microdictyon umbilicatum, and Pseudobryopsis oabuensis. The red algae consist primarily of epiphytes and turf: Anotrichium secunda, Antithamnion decipiens, Caulacanthus ustulatus, Ceramium borneense, Gayliella fimbriata, Gelidiopsis variabilis, Griffithsia heteromorpha, Herposiphonia delicatula, $H$. variabilis, Hypnea valentiae, Lophosiphonia prostrata, Neosiphonia sphaerocarpa, and Polysiphonia tsudana. One articulated coralline, Amphiroa valonioides, and one foliose red, Chrysymenia okamurae, were also unique to Johnston Atoll and French Frigate Shoals.

Fourteen algal species are common to the six islands and atolls: eight species of red algae (Anotrichium tenue, Ceramium macilentum, Corallophila apiculata, Gayliella flaccida, Heterosiphonia crispella, Hypnea spinella, Lomentaria hakodatensis, Polysiphonia scopulorum), two species of brown algae (Asteronema breviarticulatum and Lobophora variegata), and four species of green algae (Bryopsis pennata, Caulerpa serrulata, Dictyosphaeria cavernosa, Ulva clatbrata). The three atolls used in the comparison, Johnston, Wake, and Enewetak, have histories of being impacted by anthropogenic activities, and the three shoals and islands, French Frigate Shoals, Howland, and Baker, have histories of being rather pristine. Halimeda taenicola and Caulerpa bikinensis, first described from the Marshall Islands (Taylor 1950), represent two macroalgae conspicuous at Johnston Atoll that still remain absent from Hawaiian waters (Abbott and Huisman 2004).

The only tropical-subtropical Pacific atoll with a greater biodiversity of marine flora than the 189 species at Johnston Atoll is the much larger and better-studied Enewetak
Atoll in the Marshall Islands with 256 species (Tsuda 1987, 2002). NOAA cruises to the U.S. Pacific Remote Islands, however, are broadening our knowledge of the diversity of marine benthic algae from rarely visited islands and atolls in the Pacific.

\section{ACKNOWLEDGMENTS}

Our appreciation to Kimberly N. Page and Meghan L. Dailer for their participation in the collecting of algae from Johnston Atoll, and to Kerry L. Grimshaw (CRED) for producing the two maps. The constructive comments of two anonymous reviewers are acknowledged.

\section{Literature Cited}

Abbott, I. A. 1998. Some new species and new combinations of marine red algae from the central Pacific. Phycol. Res. 46:97-109. 1999. Marine red algae of the $\mathrm{Ha}-$ waiian Islands. Bishop Museum Press, Honolulu.

Abbott, I. A., and J. M. Huisman. 2003. New species, observations, and a list of new records of brown algae (Phaeophyceae) from the Hawaiian Islands. Phycol. Res. 51:173-185.

-2004. Marine green and brown algae of the Hawaiian Islands. Bishop Museum Press, Honolulu.

Aegegian, C. R., and I. A. Abbott. 1985. Deep water macroalgal communities: A comparison between Penguin Bank (Hawaii) and Johnston Atoll. Proc. 5th Int. Coral Reef Cong., Tahiti 5:47-50.

Amerson, A. B., Jr., and P. C. Shelton. 1976. The natural history of Johnston Atoll, central Pacific Ocean. Atoll Res. Bull. 192:1-479.

Anagnostidis, K., and J. Komárek. 1988. Modern approach to the classification system of cyanophytes. 3. Oscillatoriales. Arch. Hydrobiol. Suppl. 80:327-472.

Baker, H. K., G. M. Patterson, and M. Ikagawa. 1997. Preliminary description of Borzia elongata sp. nov., a representative of a valid genus in the Oscillatoriales. Arch. Hydrobiol. Suppl. 118:1-12. 
Brock, V., R. S. Jones, and P. Helfrich. 1965. An ecological reconnaissance on Johnston Island and the effects of dredging. Univ. Hawaii HIMB, Tech. Rep. 5:1-90.

Brock, V., W. van Heukelem, and P. Helfrich. 1966. An ecological reconnaissance on Johnston Island and the effects of dredging. Second annual report. Univ. Hawaii HIMB, Tech. Rep. 11:1-56.

Buggeln, R. G., and R. T. Tsuda. 1966. A preliminary marine algal flora from selected habitats on Johnston Atoll. Univ. Hawaii HIMB, Tech. Rep. 9:1-29.

- 1969. A record of benthic marine algae from Johnston Atoll. Atoll Res. Bull. 120:1-20.

Cho, T. O., and S. Fredericq. 2006. Two creeping Ceramium species (Ceramiaceae, Rhodophyta) from the Florida Keys: $C$. reptans sp. nov. and recircumscription of C. codii (Richards) Mazoyer. Phycologia 45:495-504.

Choi, H.-G., G. T. Kraft, H.-S. Kim, M. D. Guiry, and G. W. Saunders. 2008. Phylogenetic relationships among lineages of the Ceramiaceae (Ceramiales, Rhodophyta) based on nuclear small subunit rDNA sequence data. J. Phycol. 44:10331048.

Clarke, K. R., and R. M. Warwick. 2001. Change in marine communities: An approach to statistical analysis and interpretation. 2nd ed. PRIMER-E, Plymouth.

Coles, S. L., R. C. DeFelice, and D. Minton. 2001. Marine species survey of Johnston Atoll, central Pacific Ocean, June 2000. Bishop Mus. Tech. Rep. 19:1-59.

Desikachary, T. V. 1959. Cyanophyta. Indian Council of Agricultural Research, New Delhi.

Egerod, L. E. 1952. An analysis of the siphonous Chlorophycophyta with special reference to the Siphonocladales, Siphonales, and Dasycladales of Hawaii. Univ. Calif. Publ. Bot. 25:325-454, pls. 29-42.

Gosline, W. A. 1955. The inshore fish fauna of Johnston Island, a central Pacific atoll. Pac. Sci. 9:442-480.

Hillis, L. W. 1959. A revision of the genus Halimeda (order Siphonales). Publ. Inst. Mar. Sci. Univ. Tex. 6:321-403.
Hollenberg, G. J. 1963. A new species of Malaconema (Rhodophyta) from the Marshall Islands. Phycologia 2:169-172.

- 1968a. An account of the species of Polysiphonia of the central and western tropical Pacific Ocean. I. Oligosiphonia. Pac. Sci. 22:56-98.

1968b. An account of the species of the red alga Polysiphonia of the central and western tropical Pacific Ocean. II. Polysiphonia. Pac. Sci. 22:198-207.

. 1968c. An account of the species of the red alga Herposiphonia occurring in the central and western tropical Pacific Ocean. Pac. Sci. 22:536-559.

. 1968d. Phycological notes. III. New records of marine algae from the central tropical Pacific Ocean. Brittonia 20:74-82.

Itono, H. 1980. The genus Galaxaura (Rhodophyta) in Micronesia. Micronesica 16:1-19.

Komárek, J., and K. Anagnostidis. 1989. Modern approach to the classification system of cyanophytes. 4. Nostocales. Arch. Hydrobiol. Suppl. 82:247-345.

Kosaki, R. K. 1989. Centropyge nabackyi, a new species of angelfish from Johnston Atoll (Teleostei: Pomacanthidae). Copeia 1989:880-886.

Kosaki, R. K., R. L. Pyle, J. E. Randall, and D. K. Irons. 1991. New records of fishes from Johnston Atoll with notes on biogeography. Pac. Sci. 45:186-203.

Kraft, G. T. 2007. Algae of Australia. Marine benthic algae of Lord Howe Island and the southern Great Barrier Reef, 1. Green algae. ABRS, Canberra: CSIRO Publ., Melbourne.

Kraft, G. T., and I. A. Abbott. 2002. The anatomy of Neotenophycus ichthyosteus gen. et sp. nov. (Rhodomelaceae, Ceramiales), a bizarre red algal parasite from the central Pacific. Eur. J. Phycol. 37:269-278.

Littler, D. S., and M. M. Littler. 2003. South Pacific reef plants: A divers' guide to the plant life of South Pacific coral reefs. Offshore Graphics, Inc., Washington, D.C.

Lobel, P. S. 2003. Marine life of Johnston Atoll, central Pacific Ocean. Natural World Press, Inc., Vida, Oregon.

Maragos, J. E., and P. L. Jokiel. 1986. Reef 
corals of Johnston Atoll: One of the world's most isolated reefs. Coral Reefs 4:141-150.

Meñez, E. G., and H. P. Calumpong. 1982. The genus Caulerpa from central Visayas, Philippines. Smithson. Contrib. Mar. Sci. 17:1-21.

Millar, A. J. K., and D. W. Freshwater. 2005. Morphology and molecular phylogeny of the marine algal order Gelidiales (Rhodophyta) from New South Wales, including Lord Howe and Norfolk islands. Aust. Syst. Bot. 18:215-263.

Moul, E. T. 1964. New records of Halimeda and Udotea for the Pacific area. Atoll Res. Bull. 106:1-10.

Oosting, H. J. 1956. The study of plant communities. W. H. Freeman and Co., San Francisco.

Preskitt, L. B., P. S. Vroom, and C. M. Smith. 2004. A rapid ecological assessment (REA) quantitative survey method for benthic algae using photoquadrats with scuba. Pac. Sci. 58:201-209.

Randall, J. E., P. S. Lobel, and E. H. Chave. 1985. Annotated checklist of the fishes of Johnston Island. Pac. Sci. 39:24-80.

Saito, Y. 1969. The algal genus Laurencia from the Hawaiian Islands, the Philippine Islands and adjacent areas. Pac. Sci. 23:148-160.

Santelices, B. 1998. Taxonomic review of the species of Pterocladia (Gelidiales, Rhodophyta). J. Appl. Phycol. 10:237-252.

Silva, P. C., P. W. Bassom, and R. L. Moe. 1996. Catalogue of the benthic marine algae of the Indian Ocean. University of California Press, Berkeley.

Skelton, P. A., and G. R. South. 2007. The benthic marine algae of the Samoan Archipelago, South Pacific, with emphasis on the Apia District. Nova Hedwigia 132:1350.

South, G. R., and P. A. Skelton. 2000. A review of Ceramium (Rhodophyceae, Ceramiales) from Fiji and Samoa, South Pacific. Micronesica 33:45-98.

Taylor, W. R. 1950. Plants of Bikini and other northern Marshall Islands. University of Michigan Studies, Scientific Series XVIII, Ann Arbor.
Tilden, J. 1910. Minnesota algae. Vol. 1. The Myxophyceae of North America and adjacent regions including Central America, Greenland, Bermuda, the West Indies and Hawaii. Report of the Survey, Botanical Series VIII, Minneapolis.

Tsuda, R. T. 1972. Marine benthic algae of Guam. I. Phaeophyta. Micronesica 8:87115 .

1987. Marine benthic algae of Enewetak Atoll. Pages 1-9 in D. M. Devaney, E. S. Reese, B. L. Burch, and P. Helfrich, eds. The natural history of Enewetak Atoll. Vol. 2. Biogeography and systematics. Office of Scientific and Technical Information, U.S. Department of the Interior, Washington, D.C.

. 2002. Checklist and bibliography of the marine benthic algae from the Marshall Islands. Univ. Guam Mar. Lab., Tech. Rep. 106:1-34.

Tsuda, R. T., I. A. Abbott, and K. B. Foster. 2006. Marine benthic algae from Wake Atoll. Micronesica 38:207-219.

Tsuda, R. T., J. R. Fisher, P. S. Vroom, and I. A. Abbott. 2010. New records of subtidal benthic marine algae from Wake Atoll, central Pacific. Bot. Mar. 53:19-29.

Tsuda, R. T., and G. Trono Jr. 1968. Marine benthic algae from Howland Island and Baker Island, central Pacific. Pac. Sci. 22:194-197.

Tsuda, R. T., P. S. Vroom, I. A. Abbott, J. R. Fisher, and K. B. Foster. 2008. Additional marine benthic algae from Howland and Baker islands, central Pacific. Pac. Sci. 62:271-290.

Verbruggen, H., C. Vlaeminck, T. Sauvage, A. R. Sherwood, F. Leliaeri, and O. De Clerck. 2009. Phylogenetic analysis of Pseudochlorodesmis strains reveals cryptic diversity above the family level in the siphonous green algae (Bryopsidales, Chlorophyta). J. Phycol. 45:726-731.

Vroom, P. S., K. N. Page, K. A. Peyton, and J. Kanekoa Kukea-Shultz. 2006. Marine algae of French Frigate Shoals, Northwestern Hawaiian Islands: Species list and biogeographic comparisons. Pac. Sci. 60:81-95.

Wynne, M. J., I. R. Price, and D. L. Ballan- 
tine. 1989. Distinction between Hypoglossum barbatum Okamura, H. minimum Yamada and $H$. simulans sp. nov. (Delesseriaceae, Rhodophyta). Phycologia 28:28-38.

\section{Appendix 1}

\section{NOAA 2004 and 2006 Collection Sites}

JOH-01-04 (16 $\left.44.394^{\prime} \mathrm{N}, 169^{\circ} 32.073^{\prime} \mathrm{W}\right)$, lagoon, patch reef $\mathrm{N}$ of Johnston I. near dredged channel, $1.5-7.0 \mathrm{~m}$ deep, leg. P. S. Vroom and K. N. Page, I12-04 (віSн 735038-735052).

JOH-01-06, see JOH-01-04 for location and habitat, 1.5-7.0 m deep, leg. P. S. Vroom and M. L. Dailer, I-18-06 (віSн 735310-735321).

JOH-02-04 (16 $\left.44.979^{\prime} \mathrm{N}, 169^{\circ} 30.676^{\prime} \mathrm{W}\right)$, lagoon, patch reef $\mathrm{N}$ of Sand I., 4.3-7.3 m deep, leg. P. S. Vroom and K. N. Page, I-12-04 (віsн 735053735069).

JOH-02-06, see JOH-02-04 for location and habitat, 4.3-7.3 m deep, leg. P. S. Vroom and M. L. Dailer, I-18-06 (віSн 735322-735327).

JOH-03-04 (16 $\left.{ }^{\circ} 46.225^{\prime} \mathrm{N}, 169^{\circ} 28.901^{\prime} \mathrm{W}\right)$, lagoon reef NE side of atoll, 7.3-12.8 m deep, leg. P. S. Vroom and K. N. Page, I-13-04 (віSн 735070735091, 735541).

JOH-03-06, see JOH-03-04 for location and habitat, 8.2-13.4 m deep, leg. P. S. Vroom and M. L. Dailer, I-18-06 (BISH 735328-735341).

JOH-04-04 (16 $\left.{ }^{\circ} 45.518^{\prime} \mathrm{N}, 169^{\circ} 29.728^{\prime} \mathrm{W}\right)$, lagoon reef near North (Akau) I., 2.1-5.5 m deep, leg. P. S. Vroom and K. N. Page, I-13-04 (віsн 735092$735110)$.

JOH-04-06, see JOH-04-04 for location and habitat, 6.7-7.9 m deep, leg. P. S. Vroom and M. L. Dailer, I-22-06 (віSн 735342-735348).

JOH-05P-04 (16 $\left.{ }^{\circ} 45.607^{\prime} \mathrm{N}, 169^{\circ} 30.687^{\prime} \mathrm{W}\right)$, lagoon reef SE of North (Akau) I. near mooring buoy, 4.6$9.1 \mathrm{~m}$ deep, leg. P. S. Vroom and K. N. Page, I-13-04 (вISH 735111-735129, 735543).

JOH-05P-06, see JOH-05P-04 for location and habitat, 9.4-14.0 $\mathrm{m}$ deep, leg. P. S. Vroom and M. L. Dailer, I-22-06 (віsн 735349-735361).

JOH-06-04 (16 $\left.{ }^{\circ} 41.888^{\prime} \mathrm{N}, 169^{\circ} 29.092^{\prime} \mathrm{W}\right)$, lagoon reef on $\mathrm{S}$ ocean-facing side of atoll, $14.9-17.1 \mathrm{~m}$ deep, leg. P. S. Vroom and K. N. Page, I-14-04 (віSн 735130-735161, 735539).

JOH-06-06, see JOH-06-04 for location and habitat, 15.8-17.4 m deep, leg. P. S. Vroom and M. L. Dailer, I-20-06 (віSн 735362-735381).

JOH-07-04 (16 $\left.{ }^{\circ} 42.688^{\prime} \mathrm{N}, 169^{\circ} 28.759^{\prime} \mathrm{W}\right)$, lagoon reef on $\mathrm{S}$ ocean-facing side of atoll, $9.4-17.1 \mathrm{~m}$ deep, leg. P. S. Vroom and K. N. Page, I-14-04 (BISH 735162-735196).

JOH-07-06, see JOH-07-04 for location and habitat, 9.8-14.3 m deep, leg. P. S. Vroom and M. L. Dailer, I-20-06 (віSн 735382-735393).

JOH-08-04 (16 $\left.{ }^{\circ} 43.934^{\prime} \mathrm{N}, 169^{\circ} 28.978^{\prime} \mathrm{W}\right)$, leeward lagoon reef $\mathrm{S}$ of East (Hikina) I., 4.3-7.0 m deep, leg. P. S. Vroom and K. N. Page, I-14-04 (віSн 735197-735229, 735544).

JOH-08-06, see JOH-08-04 for location and habitat, 4.0-9.4 m deep, leg. P. S. Vroom and M. L. Dailer, I-20-06 (віSн 735394-735419, 735546, 735548).

JOH-09-04 (16 $\left.43.727^{\prime} \mathrm{N}, 169^{\circ} 29.115^{\prime} \mathrm{W}\right)$, leeward lagoon patch reef $\mathrm{S}$ of East (Hikina) I., 7.3-11.0 m deep, leg. P. S. Vroom and K. N. Page, I-15-04 (вISH 735196, 735230-735249, 735545).

JOH-09-06, see JOH-09-04 for location and habitat, 7.9-10.7 m deep, leg. P. S. Vroom and M. L. Dailer, I-21-06 (віSн 735420-735434, 735538).

JOH-10P-04 (16 $\left.{ }^{\circ} 45.815^{\prime} \mathrm{N}, 169^{\circ} 30.706^{\prime} \mathrm{W}\right)$, lagoon reef $\mathrm{E}$ of North (Akau) I. under white mooring buoy, 12.2-14.9 $\mathrm{m}$ deep, leg. P. S. Vroom and K. N. Page, I-15-04 (віsн 735250-735269, 735540).

JOH-10P-06, see JOH-10P-04 for location and habitat, 13.1-15.5 m deep, leg. P. S. Vroom and M. L. Dailer, I-22-06 (віSн 735435-735450).

JOH-11-04 (16 $\left.43.324^{\prime} \mathrm{N}, 169^{\circ} 31.436^{\prime} \mathrm{W}\right)$, lagoon reef S of Johnston I., 9.8-11.9 $\mathrm{m}$ deep, leg. P. S. Vroom and K. N. Page, I-16-04 (BISH 735270735290, 735542).

JOH-11-06, see JOH-11-04 for location and habitat, 6.4-10.0 m deep, leg. P. S. Vroom and M. L. Dailer, I-21-06 ( ВISH 735451-735460).

JOH-12-04 (16 $\left.{ }^{\circ} 44.860^{\prime} \mathrm{N}, 169^{\circ} 31.441^{\prime} \mathrm{W}\right)$, lagoon patch reef $\mathrm{N}$ of navigational channel, $7.9-12.2 \mathrm{~m}$ deep, leg. P. S. Vroom and K. N. Page, I-16-04 (вISH 735292-735309).

JOH-12-06, see JOH-12-04 for location and habitat, 3.6-10.7 m deep, leg. P. S. Vroom and M. L. Dailer, I-21-06 ( ВISH 735461-735471).

JOH-14-06 $\left(16^{\circ} 46.213^{\prime} \mathrm{N}, 169^{\circ} 31.117^{\prime} \mathrm{W}\right)$, forereef $\mathrm{N}$ of Johnston I., 15.2-16.8 m deep, leg. P. S. Vroom and M. L. Dailer, I-19-06 (віsн 735472-735483).

JOH-15-06 (16 $\left.47.029^{\prime} \mathrm{N}, 169^{\circ} 29.404^{\prime} \mathrm{W}\right)$, forereef NE of North (Akau) I., 15.8-16.8 m deep, leg. P. S. Vroom and M. L. Dailer, I-19-06 (віsн 735484$735495,735547)$.

JOH-16-06 (16 $\left.47.297^{\prime} \mathrm{N}, 169^{\circ} 28.435^{\prime} \mathrm{W}\right)$, forereef close to northern pass, $15.2-16.8 \mathrm{~m}$ deep, leg. P. S. Vroom and M. L. Dailer, I-19-06 (віsн 735496735504)

JOH-17-06 $\left(16^{\circ} 41.779^{\prime} \mathrm{N}, 169^{\circ} 33.639^{\prime} \mathrm{W}\right)$, forereef on extreme $W$ side of atoll, 13.7-15.8 m deep, leg. P. S. Vroom and M. L. Dailer, I-23-06 (вISH 735505-735510).

JOH-18-06 (16 $\left.43.866^{\prime} \mathrm{N}, 169^{\circ} 32.368^{\prime} \mathrm{W}\right)$, shallow lagoon reef at $\mathrm{N}$ edge of Johnston I., 1.5-2.7 m deep, leg. P. S. Vroom and M. L. Dailer, I-23-06 (вISH 735511-735524).

JOH-19-06 (16 $\left.44.683^{\prime} \mathrm{N}, 169^{\circ} 32.181^{\prime} \mathrm{W}\right)$, lagoon, backreef, 3.4-4.9 $\mathrm{m}$ deep, leg. P. S. Vroom and M. L. Dailer, I-23-06 (візн 735525-735537).

\section{Appendix 2}

Alphabetized Listing and References of Previously Recorded Algal Species Not Found in the 2004 and 2006 NOAA Collections from Johnston Atoll 
Cyanophyceae

Blennothrix lyngbyacea (Kützing) Anagnostidis \& Komárek; Buggeln and Tsuda (1969) as Hydrocoleum lyngbyaceum Gomont.

Borzia elongata Baker, Patterson \& Ikagawa; Baker et al. (1997).

Calotbrix crustacea Thuret; Buggeln and Tsuda (1969).

Calotbrix scopulorum (Weber \& Mohr) C. Agardh; Buggeln and Tsuda (1969).

Entophysalis deusta (Meneghini) Drouet \& Daily; Buggeln and Tsuda (1969).

Hormothamnium enteromorphoides Grunow; Buggeln and Tsuda (1969).

Isactis plana (Harvey) Thuret; Buggeln and Tsuda (1969).

Lyngbya aestuarii (Mertens) Liebman; Buggeln and Tsuda (1969).

Lyngbya lutea (C. Agardh) Areschoug; Buggeln and Tsuda (1969).

Lyngbya majuscula (Dillwyn) Harvey; Buggeln and Tsuda (1969).

Microcoleus chthonoplastes (Mertens) Zanardini; Buggeln and Tsuda (1969).

Microcoleus vaginatus (Vaucher) Gomont; Buggeln and Tsuda (1969).

Microcystis dimidiata (Kützing) P. Silva; Buggeln and Tsuda (1969) as Anacystis dimidiata (Kützing) Drouet \& Daily

Phormidium nigroviride (Thwaites) Anagnostidis \& Komárek; Buggeln and Tsuda (1969) as Oscillatoria nigroviridis Thwaites.

Phormidium submembranaceum (Ardissone \& Strafforello) Gomont; Buggeln and Tsuda (1969).

Schizothrix calcicola (C. Agardh) Gomont; Buggeln and Tsuda (1969).

Schizothrix tenerrima (Gomont) Drouet; Buggeln and Tsuda (1969) as Microcoleus tenerrimus Gomont.

Spirulina tenerrima Kützing; Buggeln and Tsuda (1969).

Symploca atlantica Gomont; Buggeln and Tsuda (1969).

Rhodophyceae

Callithamnion marshallense Dawson; Buggeln and Tsuda (1969).

Caloglossa leprieurii (Montagne) J. Agardh; Buggeln and Tsuda (1969).

Centroceras clavulatum (C. Agardh) Montagne; Buggeln and Tsuda (1969); Coles et al. (2001), BISH 664307.

Ceramium aduncum Nakamura; Coles et al. (2001), BISH 665241.

Ceramium borneense Weber-van Bosse; Coles et al. (2001), BISH 665773.

Ceramium maryae Weber-van Bosse; Buggeln and Tsuda (1969).

Ceramium serpens Setchell \& Gardner; Coles et al. (2001), віsн 664316.

Ceramium zacae Setchell \& Gardner; Buggeln and Tsuda (1969).

Chroodactylon ornatum (C. Agardh) Basson; Buggeln and Tsuda (1969) as Asterocystis ornata (C. Agardh) Hamel.

Dasya anastomosans (Weber-van Bosse) Wynne; Buggeln and Tsuda (1969) as Dasya adhaerens Yamada.
Dasya iridescens (Schlech) Millar \& Abbott; Coles et al. (2001), вISH 665267.

Dasya murrayana Abbott \& Millar; Coles et al. (2001), BISH 664332.

Dasya sinicola (Setchell \& N. L. Gardner) Dawson; Buggeln and Tsuda (1969).

Diplothamnion jolyi van den Hoek; Coles et al. (2001), BISH 665768.

Gayliella fimbriata (Setchell \& N. L. Gardner) T. O. Cho \& S. M. Boo; Buggeln and Tsuda (1969) as Ceramium fimbriatum Setchell \& Gardner.

Gelidiopsis variabilis (J. Agardh) Schmitz; Coles et al. (2001), BISH 664287.

Gelidium crinale (Turner) Gaillon; Buggeln and Tsuda (1969).

Gelidium pusillum (Stackhouse) Le Jolis; most likely misidentified: Gelidium isabelae W. R. Taylor (Millar and Freshwater 2005) or Pterocladiella caerulescens (Kützing) Santelices (Skelton and South 2007).

Griffithsia metcalfii C. K. Tseng; Buggeln and Tsuda (1969).

Griffithsia ovalis Harvey; Buggeln and Tsuda (1969).

Griffithsia schousboei Montagne; Coles et al. (2001), BISH 665766.

Herposiphonia arcuata Hollenberg; Coles et al. (2001), BISH 664301.

Herposiphonia crassa Hollenberg; Coles et al. (2001), BISH 665829.

Herposiphonia delicatula Hollenberg; Coles et al. (2001), BISH 665226.

Hypnea pannosa J. Agardh; Coles et al. (2001), вІSн 665258.

Hypnea valentiae (Turner) Montagne; Buggeln and Tsuda (1969) as Hypnea esperi Bory; Coles et al. (2001), віsн 664303.

fania adhaerens Lamouroux; Coles et al. (2001), вISH 665271.

Kallymenia sp.; Coles et al. (2001), віsн 665239.

Lophosiphonia prostrata (Harvey) Falkenberg; Coles et al. (2001), вISн 665813.

Monosporus indicus Børgesen; Coles et al. (2001), вІsн 665791.

Neosiphonia howei (Hollenberg in Taylor) Skelton \& South; Coles et al. (2001) as Polysiphonia bowei Hollenberg, вISH 665248.

Neotenophycus ichthyosteus Kraft \& Abbott (2002).

Peyssonnelia conchicola Piccone \& Grunow; Coles et al. (2001), вISH 664338.

Polysiphonia triton Silva; Hollenberg (1968a) (holotype) as Polysiphonia tenuis Hollenberg (replaced name).

Wurdemannia sp.; Buggeln and Tsuda (1969).

Phaeophyceae

Asteronema breviarticulatum (J. Agardh) Ouriques \& Bouzon; Buggeln and Tsuda (1969) as Ectocarpus breviarticulatus J. Agardh.

Dictyota bartayresiana Lamouroux; Aegegian and Abbott (1985), $118 \mathrm{~m}$ deep.

Feldmannia irregularis (Kützing) Hamel; Buggeln and Tsuda (1969) as Ectocarpus irregularis Kützing.

Hincksia indica (Sonder) J. Tanaka; Buggeln and Tsuda (1969) as Ectocarpus indicus Sonder. 
Sphacelaria tribuloides Meneghini; Buggeln and Tsuda (1969); Coles et al. (2001), віsн 665790.

Chlorophyceae

Palmogloea protuberans (J. E. Smith) Kützing; Buggeln and Tsuda (1969), most likely misidentified because $P$. protuberans is a freshwater species.

Ulotbrix flacca (Dillwyn) Thuret; Coles et al. (2001) as Ulotbrix pseudoflacca Wille, вISн 665266.

Ulvophyceae

Ulva kylinii (Bliding) Hayden, Blomster, Maggs, P. C. Silva, M. J. Stanhope \& J. R. Waaland; Buggeln and Tsuda (1969) as Enteromorpha kylinii Bliding.

Cladophorophyceae

Boodlea composita (Harvey) Brand; Buggeln and Tsuda (1969).

Cladophora crystallina (Roth) Kützing; Buggeln and Tsuda (1969).

Dictyosphaeria cavernosa (Forsskål) Børgesen; Coles et al. (2001), віsн 664346.

Bryopsidophyceae

Caulerpa lentillifera J. Agardh; Coles et al. (2001), віsн 665249.

Caulerpa urvilleana Montagne; Buggeln and Tsuda (1969).

Caulerpella ambigua (Okamura) Prud'homme van Reine \& Lokhorst; Buggeln and Tsuda (1969) and Aegegian and Abbott (1985) as Caulerpa ambigua Okamura, $70 \mathrm{~m}$ deep; Coles et al. (2001) as Caulerpa ambigua, вISн 664309.
Codium arabicum Kützing; Buggeln and Tsuda (1969).

Derbesia marina (Lyngbye) Solier; Buggeln and Tsuda (1969).

Halimeda discoidea Decaisne; Buggeln and Tsuda (1969); Coles et al. (2001), віsн 663190.

Halimeda gracilis Harvey ex J. Agardh; Aegegian and Abbott (1985), 50-125 (136) m deep.

Halimeda opuntia (Linnaeus) Lamouroux; Coles et al. (2001), віSн 665799.

Halimeda tuna (Ellis \& Solander) Lamouroux; Moul (1964); Buggeln and Tsuda (1969).

Ostreobium quekettii Bornet \& Flahault; Buggeln and Tsuda (1969) as Ostreobium reineckei Bornet.

Pseudobryopsis oabuensis Egerod; Coles et al. (2001) as Trichosolen oabuensis (Egerod) Taylor, віsн 664296.

Dasycladophyceae

Parvocaulis clavatus (Yamada) S. Berger, Fettweiss, Gleissberg, Liddle, U. Richter, Sawitzky \& Zuccarello; Buggeln and Tsuda (1969) as Acetabularia clavata Yamada.

Parvocaulis exiguus (Solms-Laubach) S. Berger, Fettweiss, Gleissberg, Liddle, U. Richter, Sawitzky \& Zuccarello; Buggeln and Tsuda (1969) as Acetabularia tsengiana Egerod.

Parvocaulis parvulus (Solms-Laubach) S. Berger, Fettweiss, Gleissberg, Liddle, U. Richter, Sawitzky \& Zuccarello; Buggeln and Tsuda (1969) as Acetabularia mobii Solms-Laubach. 
